\title{
Topical issue on nuclear symmetry energy
}

\author{
Published online: 21 February 2014 - (C) Società Italiana di Fisica / Springer-Verlag 2014
}

The nuclear symmetry energy, which encodes the energy related to neutron-proton (isospin) asymmetry in the equation of state of nuclear matter, and in particular its density dependence, $E_{\mathrm{sym}}(\rho)$, is a crucial ingredient necessary to resolve many important issues in both nuclear physics and astrophysics, such as the structures of rare isotopes and neutron stars, dynamics of heavy-ion reactions and supernovae explosions, emissions of neutrinos from protoneutron stars and gravitational waves from spiraling neutron star binaries.

The density dependence of the nuclear symmetry energy has been studied extensively within both phenomenological approaches and microscopic many-body theories using almost all available effective and/or realistic nuclear interactions. Both methods have their respective advantages and disadvantages as well as application ranges. Despite the significant progress made in recent years especially in constraining the nuclear symmetry energy around saturation density, there are still many interesting theoretical issues to be solved especially at both very low densities, where cluster formation becomes important, and supra-saturation densities, where many-body forces and short-range correlations are critical but poorly known. Thus, the lack of a more precise theoretical determination of $E_{\text {sym }}(\rho)$ is mostly due to our limited knowledge about the in-medium spin and isospin dependence of nuclear forces and correlations besides technical difficulties of accurately treating quantum many-body systems.

Information on the density dependence of the nuclear symmetry energy can be extracted from both terrestrial nuclear laboratory experiments and astrophysical observations. Comparisons of measurements to model predictions have provided important means to explore the nuclear symmetry energy. A number of observables, both in nuclear dynamics and structure experiments, have been found to be sensitive to $E_{\mathrm{sym}}(\rho)$ at various densities. These include binding energies, neutron-skins, giant and pygmy resonances in nuclei, as well as isospin-asymmetric yield ratios of nucleons, mirror nuclei and mesons, such as $n / p, t /{ }^{3} \mathrm{He}, \pi^{-} / \pi^{+}$and $K^{+} / K^{0}$, differential collective flows of nucleons and light mirror nuclei, reaction-time scales measured using two-particle correlation functions in heavy-ion collisions at low-to-intermediate energies, etc. Analyses of experimental data of these observables have allowed the community to significantly constrain the nuclear symmetry energy around and below the saturation density. In particular, the obtained results are mostly consistent with a magnitude of the nuclear symmetry energy around $30 \mathrm{MeV}$ and a density slope around $60 \mathrm{MeV}$ at saturation density. However, many of the analyses carried out so far lack quantified theoretical uncertainties. Moreover, some of the experimental data also suffer from large uncertainties. Most of them reside in difficulties in precisely determining global features of the collision event (impact parameter, reaction plane, etc.) on an event-by-event basis, thus making it difficult to perform unambiguous comparisons to microscopic models of heavyion collisions. Terrestrial observables sensitive to the high-density behavior of the nuclear symmetry energy are less known and there are few experimental data available. A few analyses done so far using limited data available have drawn conflicting conclusions about the nuclear symmetry energies at supra-saturation densities. Looking forward, it is exciting to note that dedicated new detectors are being built to better constrain the nuclear symmetry energy in a broad density range in several laboratories. In particular, it is worth noting that powerful experimental techniques, including the development of new experimental setups covering large solid angles and characterized by high energy, timing, angular and isotopic resolution over a wide range of particle rapidities, are key to resolve still existing experimental uncertainties. Some of these developments require special investments in the development of new detectors and digital electronics facilities that can be used in large-scale experiments. Important opportunities are also being offered by the advanced exotic beam facilities permitting studies of reactions with very high isospin asymmetries, thus enhancing the 
observable effects induced by the density dependence of the nuclear symmetry energy. On the other hand, compact stellar objects and astrophysical events, such as neutron stars, open a window into both the bulk and microscopic properties of nuclear matter at extreme densities and isospin asymmetries. Various astrophysical observables, most noticeably the radii, the frequencies of torsional oscillations, the r-mode instability window of neutron stars, the neutrino flux from supernovae explosions and the cooling curves of protoneutron stars, have been employed to constrain the nuclear symmetry energy. Generally, the extracted value of the nuclear symmetry energy around saturation density is consistent with that obtained from studying terrestrial nuclear experiments although some of the analyses can only give an upper or lower limit. However, because of the well-known difficulties in obtaining accurate values of some of these observables, especially the radii of neutron stars, much more data and analyses are needed before mapping out accurately the complete density dependence of the nuclear symmetry energy using astrophysical observations. Thanks to the recent investment by ESA and NASA, exciting new opportunities exist for better constraining the nuclear symmetry energy using X-ray data from missions such as Chandra, XMM-Newton and the new NICER (Neutron Star Interior Composition Explorer) as well as the Gamma-ray data from the Fermi observatory.

Despite the many remaining challenges in determining completely the density dependence of the nuclear symmetry energy, our community has made impressive progress in solving many difficult problems in this field. Theories and experiments/observations go hand in hand and our achievements in studying the nuclear symmetry energy are the results of strong collaborations between experimentalists and theorists around the world. Certainly, future progress relies on the continuation of these collaborations of scientists from both nuclear physics and astrophysics communities.

Recognizing the importance of this topic, the Editorial Board of The European Physical Journal A (EPJA) found it is the right time to invite the experts to review, analyze and summarize in this Topical Issue on the Nuclear Symmetry Energy the recent progress and most importantly, to identify and develop effective strategies to explore both theoretically and experimentally the remaining open questions in this field. Realizing that various interesting works on the nuclear symmetry energy are being carried out by many experts around the world, we aimed at providing a representative overview of the copious research in this field and apologize to those groups whose results are not included in this special issue. The 40 articles of the issue are organized according to four different topics in the following way:

\section{Open theoretical issues on nuclear symmetry energy}

- S. Gandolfi et al., The equation of state of neutron matter, symmetry energy and neutron star structure.

- K. Hebeler, A. Schwenck, Symmetry energy, neutron skin, and neutron star radius from chiral effective field theory interactions.

- Wei Zuo et al., Three-body force effect on nuclear symmetry energy and single-particle properties of asymmetric nuclear matter.

- A. Carbone et al., Tensor force effects and high-momentum components in the nuclear symmetry energy.

- Hyun Kyu Lee, Mannque Rho, Topology change and tensor forces for the EoS of dense baryonic matter.

- Y. Seo, S.-J. Sin, Symmetry energy from holographic QCD.

- Kie Sang Jeong, Su Houng Lee, Symmetry energy from QCD sum rules.

- S. Typel et al., Effects of the liquid-gas phase transition and cluster formation on the symmetry energy.

- J. Margueron et al., Effect of pairing on the symmetry energy and the incompressibility.

- B.K. Agrawal et al., Symmetry energy of warm nuclear systems.

- W. Nazarewicz et al., Symmetry energy in nuclear density functional theory.

- Chang Xu et al., Relationship between the symmetry energy and the single-nucleon potential in isospin-asymmetric nucleonic matter.

- F. Sammaruca, Microscopic approach to the nucleon-nucleon effective interaction and nucleon-nucleon scattering in symmetric and isospin-asymmetric nuclear matter.

- R.J. Charity et al., Isospin dependence of nucleon correlations in ground-state nuclei.

- Ad.R. Raduta, Clusterized nuclear matter in the (proto-)neutron star crust and the symmetry energy.

\section{Constrains on the nuclear symmetry energy from terrestrial nuclear laboratory experiments}

- J. Piekarewicz, Symmetry energy constraints from giant resonances: A relativistic mean-field theory overview.

- G. Colò et al., Symmetry energy from the nuclear collective motion: constraints from dipole, quadrupole, monopole and spin-dipole resonances.

- X. Viñas et al., Density dependence of the symmetry energy from neutron skin thickness in finite nuclei. 
- A. Tamii et al., Electric dipole response of ${ }^{208} \mathrm{~Pb}$ from proton inelastic scattering: Constraints on neutron skin thickness and symmetry energy.

- L.-W. Chen et al., Probing isospin- and momentum-dependent nuclear effective interactions in neutron-rich matter.

- Maria Colonna et al., Theoretical predictions of experimental observables sensitive to the symmetry energy.

- Z. Kohley, S.J. Yennello, Heavy-ion collisions: Direct and indirect probes of the density and temperature dependence of $E_{\mathrm{sym}}$.

- E. De Filippo, A. Pagano, Experimental effects of dynamics and thermodynamics in nuclear reactions on the symmetry energy as seen by the CHIMERA $4 \pi$ detector.

- G. Ademard et al., Isospin effects and symmetry energy studies with INDRA.

- Dao T. Khoa et al., Folding model study of the charge-exchange scattering to the isobaric analog state and implication for the nuclear symmetry energy.

- A.B. McIntosh et al., How much cooler would it be with some more neutrons?

- S. Hudan, R.T. deSouza, Timescale for isospin equilibration in projectile breakup.

- Zhi-Gang Xiao et al., Probing nuclear symmetry energy at high densities using pion, kaon, eta and photon productions in heavy-ion collisions.

- P. Russotto et al., Flow probe of symmetry energy in relativistic heavy-ion reactions.

- K. Hagel et al., The equation of state and symmetry energy of low-density nuclear matter.

Imprints and extraction of nuclear symmetry energy on/from astrophysical observations

- James M. Lattimer, Andrew W. Steiner, Constraints on the symmetry energy using the mass-radius relation of neutron stars.

- W.G. Newton et al., Contraints on the symmetry energy from observational probes of the neutron star crust.

- Kei Iida, Kazuhiro Oyamatsu, Symmetry energy, unstable nuclei and neutron star crusts.

- J.M. Pearson et al., Symmetry energy: nuclear masses and neutron stars.

- Constança Providência et al., Imprint of the symmetry energy on the inner crust and strangeness content of neutron stars.

- F.J. Fattoyev et al., Probing the high-density behavior of symmetry energy with gravitational waves.

- Tobias Fischer et al., Symmetry energy impact in simulations of core-collapse supernovae.

Future perspectives: new experiments and detectors for studying nuclear symmetry energy in terrestrial laboratories

- R. Bougault for the FAZIA Collaboration, The FAZIA project in Europe: REDD phase.

- C.J. Horowitz et al., Electroweak measurements of neutron densities in CREX and PREX at JLab, USA.

- Byungsik Hong et al., Plan for nuclear symmetry energy experiments using the LAMPS system at the RIB facility $R A O N$ in Korea.

We hope this topical issue helps stimulating further studies on the nuclear symmetry energy and serves as a useful reference especially for young students who are just entering this field. We would like to thank all the contributors for their efforts in making this special issue possible and useful, the EPJA Editorial Board and publishers for their support and encouragement. Last but not least, we thank Ms. Monica Bonetti for her hard work and invaluable help during the preparation of this issue.

Bao-An Li (Texas A\&M University-Commerce), Àngels Ramos (Universitat de Barcelona), Giuseppe Verde (INFN, Sezione di Catania), and Isaac Vidaña (Universidade de Coimbra) 